\title{
THE INFLUENCE OF THE DEGREE OF THE RIVET HOLE SIZING ON THE FATIGUE LIFE
}

\author{
Adam Lipski \\ University of Technology and Life Sciences in Bydgoszcz \\ Faculty of Mechanical Engineering, Bydgoszcz, Poland
}

\begin{abstract}
The paper presents the results of fatigue tests of specimens with sized rivet holes. Samples for tests were made of 0.05 " (1.27 mm) thick non-clad plates of aluminium grade 2024-T3. Rivet holes were prepared assuming that they shall be used for $3 \mathrm{~mm}$ nominal diameter snap head rivets for aviation-related purposes. Different sizing degrees were achieved by drilling holes of different diameters in the samples followed by the sizing process using a sizing mandrel of the same diameter of $3.15 \mathrm{~mm}$. Holes in the test samples were drilled using special device ensuring appropriate quality and repeatability of the holes. Five different sizing degrees were achieved be means of five drills of different diameters.

Samples with holes of varying degrees of sizing were tested under constant amplitude sinusoidal loading conditions (cycle asymmetry coefficient $R=0$ ) at the load frequency of $5 \mathrm{~Hz}$. The study was conducted at three levels of maximum stress in the cycle. Fatigue life test results were presented in the form of fatigue diagrams (regression lines) determined in the bi-logarithmic coordinate system $\log N, \log S_{\max }$. The results of the fatigue life tests received for the drilled as well as drilled and reamed holes were also presentedfor comparison. The results obtained lead to the conclusion that that the lowest fatigue life characterises samples with drilled holes and drilled and reamed holes. Fatigue life of specimens with holes for rivets improved (by 50\% to 74\%, depending on load level), even as a result of the hole surface polishing only (minimum sizing degree), whilst the two-fold growth of fatigue life was achieved for holes of a slight sizing degree. A further significant increase in fatigue life was achieved by the cold work of the hole's surface. Based on the location of the fatigue diagrams and the fatigue life tests results, it may be concluded that the higher a sizing degree, the higher the fatigue life growth. The growth is also proportional to the specimen load level: the lower the load level, the higher the fatigue life growth.

Fatigue diagrams obtained from tests were divided into three groups: diagrams for drilled holes and drilled and reamed holes, diagrams for holes with a low degree of sizing and diagrams for holes with a high degree of sizing. This division was confirmed by statistical tests of regression lines parallelism by the "peer-to-peer" method.
\end{abstract}

\section{INTRODUCTION}

The main elements of the typical aircraft structure are built as thin-walled structures. The individual sheets are most often connected to each other and stiffening elements by means of rivets and screws, but the former predominate. Tens of thousands, even millions of rivet holes occurring in such structures are sites of stress concentration and potentially fatigue crack initiation areas, which significantly impinges on the fatigue life of the whole structure and consequently on the safety of flight. 
Rivet holes may be subjected to treatments that enhance their resistance to fatigue cracking. The most important operations include reaming and sizing. Reaming results in less diameter scatter and smoother holes. Compressive stresses are inserted into the inner layers of the material by sizing. These stresses hinder the formation of the hole's surface fatigue cracks. The positive effect of hole sizing on fatigue life was pointed out i.e. in [1].

Sizing of the holes can be made using special burnishing heads. This technology cannot be used for sizing the holes with diameters smaller than $3 \mathrm{~mm}$. These holes can be sized by using a mandrel with an appropriate diameter. The resulting degree of deformation of the hole's surface depends on the difference between the diameter of the drilled hole and the sizing mandrel diameter.

The aim of this study is to analyse the course of the rivet hole sizing process using sizing mandrels and to evaluate the impact of the rivet hole sizing degree on the fatigue life. The research described in the study concerned improvement of the fatigue life of riveted joints as a result of local strain hardening of the rivet hole by the sizing process, which also results in improving the hole surface smoothness.

Experimental analyses of the sizing process and numerical simulations of the sizing performed using ABAQUS FEM software were presented in detail in work [2]. The analyses presented in paper [2] were extended in this work by the statistical analyses of the experimental results.

Sizing and fatigue tests were performed in the Department Laboratory for Research on Materials and Structures (certified by the Polish Centre for Accreditation - PCA AB 372) of the Faculty of Mechanical Engineering at the University of Technology and Life Sciences in Bydgoszcz, using the testing machine INSTRON 8501.

\section{TEST SAMPLES}

Samples for tests were made of $1.27 \mathrm{~mm}$ thick non-clad plates of aluminium grade 2024-T3. Rivet holes were prepared assuming that they shall be used for $3 \mathrm{~mm}$ nominal diameter snap head solid rivets for aviation-related purposes [3]. According to [4], finished holes for such rivets should be of $3.1 \mathrm{~mm}$ diameter with positive tolerance of $+0,1 \mathrm{~mm}$. Holes of such a diameter were obtained by two operations. The first one was drilling the hole and the second one - sizing the hole to the diameter of $3.1 \mathrm{~mm}$. The research covered several degrees of hole sizing. In this study, the sizing degree $k$ means the following relationship:

$$
k=\left(\frac{d_{k}-d_{w}}{d_{w}}\right) \cdot 100 \%
$$

where:

$d_{k}$ - hole diameter after sizing,

$d_{w}-$ hole diameter after drilling.

Samples with holes made in the conventional way (drilling or drilling and reaming) were also prepared for comparison purposes.

Different sizing degrees were achieved by drilling holes of different diameters in the samples followed by sizing process using a sizing mandrel of the same diameter. Holes in the test samples were drilled using a special device ensuring the appropriate quality and repeatability of the holes. Figure 1a shows the profile of the test samples and the hole diameters. Based on preliminary tests it was concluded that a $3.15 \mathrm{~mm}$ nominal diameter mandrel is necessary to size the holes to the diameter of $3.1 \mathrm{~mm}$. The shape of the sizing mandrel used is shown in Fig. 1b. Five different sizing degrees were achieved be means of five drills of different diameters for preliminary holes and one sizing mandrel. $k=6.9 \% \quad\left(d_{w}=2.9 \mathrm{~mm}\right), k=5.08 \% \quad\left(d_{w}=2.95 \mathrm{~mm}\right), \quad k=3.33 \%$ $\left(d_{w}=3.0 \mathrm{~mm}\right), k=1.64 \%\left(d_{w}=3.05 \mathrm{~mm}\right), k=0.30 \%\left(d_{w}=3.1 \mathrm{~mm}\right)$. 
a)

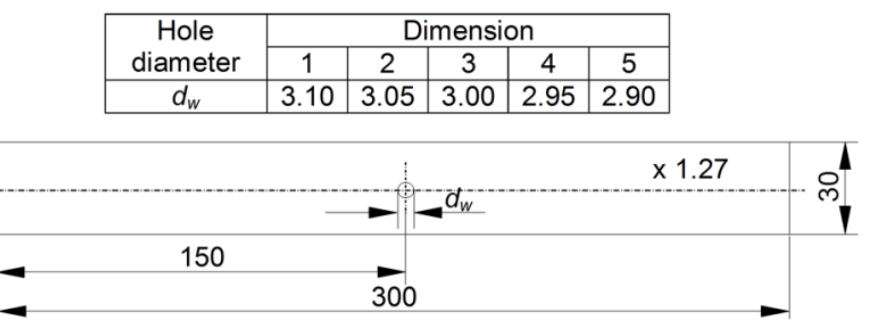

b)

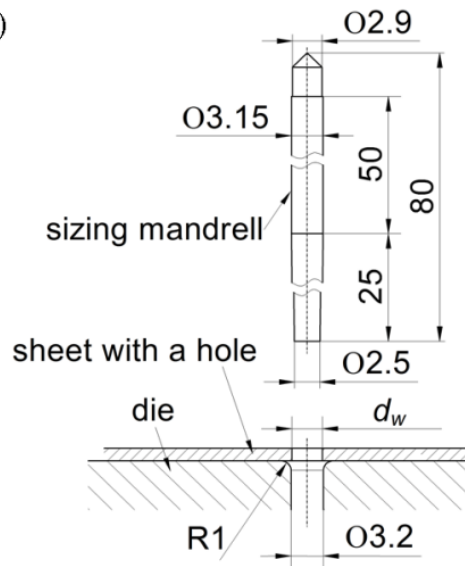

Fig. 1. Samples for fatigue tests (a) and the sizing mandrel (b)

Holes were sized using the testing machine INSTRON 8501. To reduce sample deformation in its thickness direction during the sizing process, the diameter of the hole in the die supporting the sample was slightly bigger $(\varnothing 3,2 \mathrm{~mm})$ than the mandrel diameter $(\varnothing 3,15 \mathrm{~mm})$.

Hole diameters were measured after sizing. The measurement results were presented in the form of a graph (Fig. 2), where hole diameters measured after drilling were additionally presented. Based on the prepared graphs, it can be noted that the machining-related deviation of the diameter depends on the diameter of the hole. The bigger the drill diameter, the smaller the deviation. One can conclude that despite the additional drill guide used during hole drilling, the holes are enlarged due to radial run-out.

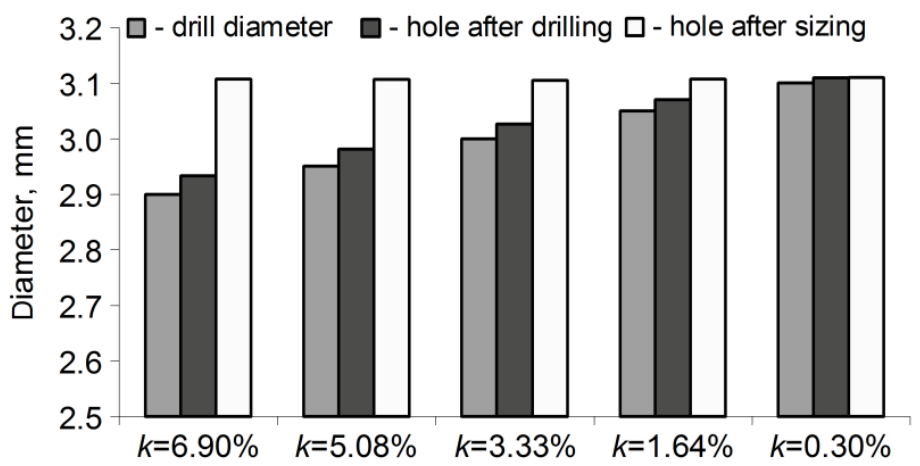

Fig. 2. The results of diameter measurement of holes in samples, acquired after drilling and after sizing

\section{FATIGUE TESTS}

The samples with holes of different sizing degree were subject to fatigue tests. The tests were performed under zero-tension cycle conditions (cycle asymmetry factor $R=0$ ) with load frequency of $5 \mathrm{~Hz}$. The tests were performed for three levels of a maximum load of the cycle $S_{\max }=150,175$ and $200 \mathrm{MPa}$ (three samples for each load level). As in the case of sizing of the holes, fatigue tests were also performed using the testing machine INSTRON 8501.

Results obtained during fatigue tests were presented in Fig. 3 in the bilogatithmic coordinate system, in the form of fatigue graphs determined assuming $S_{\max }$ as the independent variable of the stress and the fatigue life $N$ as the dependant variable. Results of the fatigue life tests acquired for drilled holes (W) as well as drilled and reamed holes (R) were also added in the figure for comparison. Fig. 4 includes the summary of the average fatigue life ratio of samples with sized holes to samples with drilled holes, presented for individual load levels. 


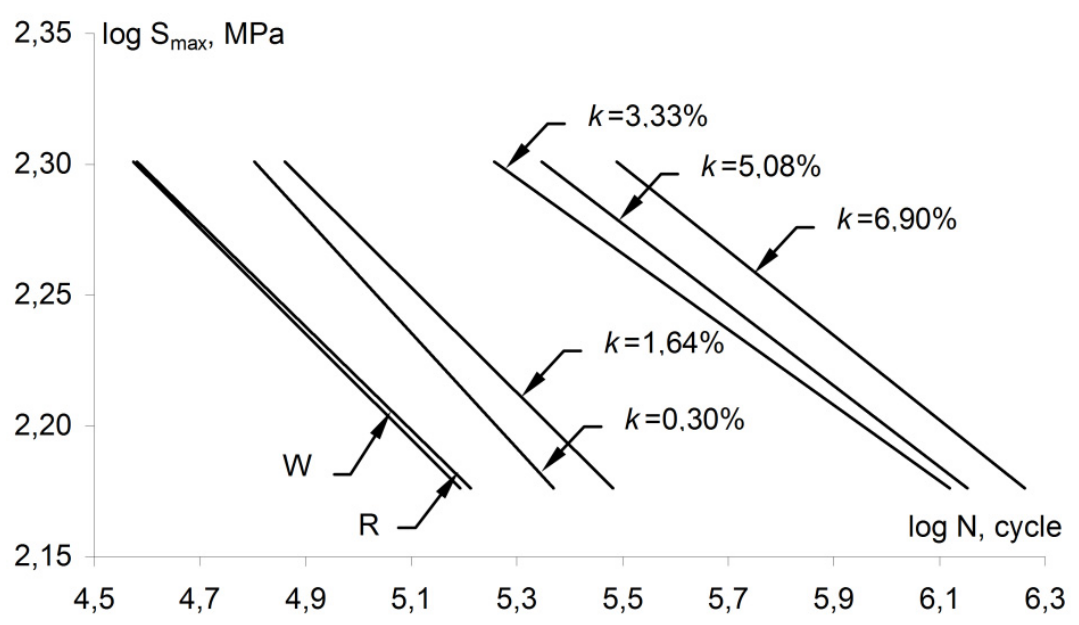

Fig. 3. Fatigue life graphs obtained for samples with holes of different sizing degree

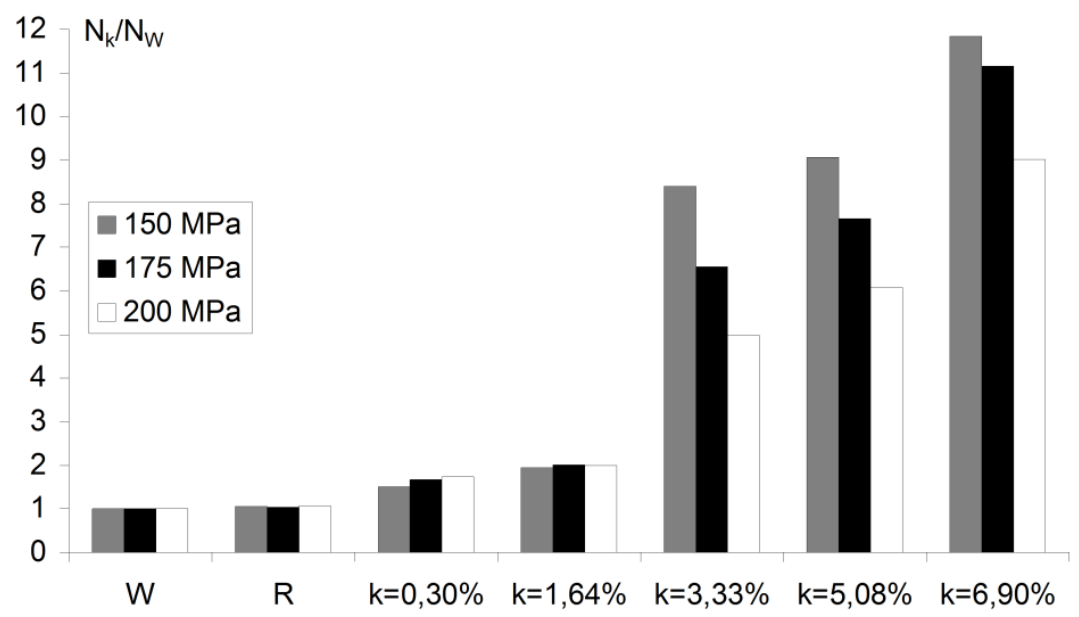

Fig. 4. Average fatigue life ratio of samples with sized holes $N_{k}$ to samples with drilled holes $N_{W}$

Based on the achieved results, one can conclude that the lowest fatigue life characterised samples with drilled holes and drilled and reamed holes. The fatigue life of riveted joints improved (by 50 to $74 \%$, depending on load level), even as a result of the hole's surface polishing only. This improvement was obtained for samples with $d_{w}=3.1 \mathrm{~mm}(k=0.30 \%)$ holes. A two-fold growth of the fatigue life was achieved for $d_{w}=3.05 \mathrm{~mm}$ hole with a slight sizing degree of $k=1.64 \%$.

A further significant increase in fatigue life was achieved by the cold work of the hole's surface and, based on the position of the fatigue life graphs obtained (Fig. 3) and fatigue life values (Fig. 4), it may be concluded that the higher the sizing degree, the higher the fatigue life growth. The growth is also proportional to a mandrel load level. For example, a five-fold fatigue life increase (for the load level of $200 \mathrm{MPa}$ ) and an eight-fold increase (for the load level of $150 \mathrm{MPa})$ were achieved for samples with $d_{w}=3.0 \mathrm{~mm}(k=3.33 \%)$ sized holes. A maximum improvement of fatigue life was obtained for samples with $d_{w}=2.9 \mathrm{~mm}(k=6.90 \%)$ sized holes, but the results were characterised by the highest dispersion. A nine-fold improvement of fatigue life was achieved for those samples compared to samples with drilled holes for the load level of $S_{\max }=200 \mathrm{MPa}$ and nearly a twelve-fold increase for the load level of $S_{\max }=150 \mathrm{MPa}$. 


\section{STATISTICAL ANALYSES OF THE EXPERIMENTAL RESULTS}

Fatigue life test results presented in Fig. 3 in the form of fatigue diagrams are regression lines determined in the bi-logarithmic coordinate system $\log N, \log S_{\max }$ as straight lines described by the equation:

$$
\log N=a \cdot \log S_{\max }+b
$$

where

$a-$ a slope of a straight line,

$b-$ a free term in an expression.

The slope of a straight line $a$, the free term in an expression $b$ and the coefficient of determination $R^{2}$ values for each line were presented in tab. 1 .

Table 1. Parameters of linear regression lines and the coefficient of determination values

\begin{tabular}{|l|l|l|l|}
\hline Sizing degree & $a$ & $b$ & $R^{2}$ \\
\hline $\mathrm{W}$ & $-5,077$ & 16,23 & 0,985 \\
\hline $\mathrm{R}$ & $-5,064$ & 16,23 & 0,992 \\
\hline$k=0.30 \%$ & $-4,530$ & 15,23 & 0,979 \\
\hline$k=1.64 \%$ & $-4,969$ & 16,29 & 0,981 \\
\hline$k=3.33 \%$ & $-6,896$ & 21,13 & 0,985 \\
\hline$k=5.08 \%$ & $-6,456$ & 20,20 & 0,983 \\
\hline$k=6.90 \%$ & $-6,182$ & 19,71 & 0,803 \\
\hline
\end{tabular}

It can be seen that there are three sets of lines. Lines for drilled holes (W) and drilled and reamed holes (R) comprise the first set. These lines have the slopes of a straight line $a$ over

the value of 5 . Lines for holes with a low degree of sizing $(k=0.30 \%, k=1.64 \%)$ are the second set. The slopes of a straight line $a$ for these lines are under the value of 5 . The values free term in an expression $b$ for the first set are close to the second set. The third set of lines for a high degree of sizing ( $k$ equal to or greater than $3.33 \%$ ) has significantly different values of $a$ and $b$ than the first and second sets.

The above observations were confirmed by the statistical tests of regression lines parallelism by the "peer-to-peer" method, but there are two sets of lines in this case.

The Students's t-test was used for this analysis. The test statistic was determined by the equation:

$$
t=\frac{a_{1}-a_{2}}{s_{a_{1}-a_{2}}}
$$

where:

$$
s_{a_{1}-a_{2}}=\sqrt{\frac{\sum_{i=1}^{n_{1}}\left(y_{i 1}-\hat{y}_{i 1}\right)^{2}+\sum_{i=1}^{n_{2}}\left(y_{i 2}-\hat{y}_{i 2}\right)^{2}}{n_{1}+n_{2}-4} \cdot\left(\frac{1}{\sum_{i=1}^{n_{1}}\left(x_{i 1}-\bar{x}_{i 1}\right)^{2}}+\frac{1}{\sum_{i=1}^{n_{2}}\left(x_{i 2}-\bar{x}_{i 2}\right)^{2}}\right)}
$$

The critical value of Student's t-test was determined for the significance level $\alpha=0.05$ and degrees of freedom $n=n_{1}+n_{2}-4=14$ ( $n_{1}=n_{2}=9$ - number of data for each lines). 
If the following equation:

$$
|t|<t_{\alpha ; n}
$$

is satisfied, then there is a reason to accept the null hypothesis about parallelism of regression lines in the statistical sense with the significance level. The calculation results were presented in tab. 2 .

Table 2. The test statistic values for parallelism of regression lines tests

\begin{tabular}{|c|c|c|c|c|c|c|}
\hline & $\begin{array}{l}k \\
6.90 \%\end{array}$ & $\begin{array}{l}k \\
5.08 \%\end{array}$ & $\begin{array}{l}k \\
3.33 \%\end{array}$ & $\begin{array}{l}=k \\
1.64 \%\end{array}$ & $=\begin{array}{l}k \\
0.30 \%\end{array}$ & $=\mathrm{R}$ \\
\hline W & 0,937 & 3,488 & 4,633 & $-0,308$ & $-1,601$ & $-0,044$ \\
\hline $\mathrm{R}$ & 0,956 & 3,834 & 5,089 & $-0,303$ & $-1,761$ & \\
\hline$k=0.30 \%$ & 1,396 & 4,748 & 5,873 & 1,207 & & \\
\hline$k=1.64 \%$ & 1,023 & 3,590 & 4,682 & & & \\
\hline$k=3.33 \%$ & $-0,596$ & $-0,978$ & & & & \\
\hline$k=5.08 \%$ & $-0,229$ & & & & & \\
\hline
\end{tabular}

The first set of lines comprises lines for drilled holes (W), drilled and reamed holes (R) and for holes with a low degree of sizing. All lines are parallel to one another from this set (in the statistical sense). The second set is formed by the lines for a high degree of sizing. The line for $k=3.33 \%$ is parallel to the line for $k=5.08 \%$. The different runs of lines for these sets result from a difference of plastic deformation value around the hole for the rivet. The line with the highest degree of sizing $(k=6.90 \%)$ is parallel to all lines in the statistical sense. It is so because the statistical dispersion is highest for this line. The minimal value of the coefficient of determination (tab. 1) indicates this.

\section{CONCLUSIONS}

Fatigue life of riveted joints improves thanks to additional preparatory operations performed prior to riveting, such as hole sizing. Results obtained in this research confirm the positive effect of hole sizing on fatigue life. Cold work and polishing of the hole's surface by the sizing mandrel hinders initiation of micro-cracks. It should be emphasized that a number of factors influence fatigue life of the sample with riveted holes.

The results of this work indicate that just the hole surface polishing by reaming or low degree sizing improves fatigue life. The optimal value of sizing degree is $k=3 \div 5 \%$ - higher value of sizing degree effectiveness in increase of the statistical dispersion of fatigue life.

This work was financed from the funds of the Polish Ministry of Science and Higher Education in the years 2006-2010 as the research project No. 61/EUR/2006/02.

\section{REFERENCES}

[1] Larouche S. at all. (2001). Influence of Cold Working and Interference Fit on Fatigue Life of 7475-T7351 Aluminum Alloy Fastener Hole. Ed. J. Rouchon Cepadues. Design for Durability in the $21^{\text {th }}$ Symposium of the International Committee on Aeronautical Fatigue, 27-29 June 2001 (pp. 681-698), Toulouse, France.

[2] Lipski A., Mroziński S., Lis Z. (2011). Evaluation of the rivet hole sizing degree effect on the fatigue life, Journal of Polish CIMAC, Vol. 6(3), pp. 119-125.

[3] Branch standard: Snap head rivet for aircraft. BN-70/1121-03 (in Polish).

[4] Branch standard: Rivets for aviation-related purposes. Length selection, formed rivet heads dimensions and diameters of holes for rivets. BN-70/1120-03 (in Polish). 